\title{
Autophagy facilitates multidrug resistance development through inhibition of apoptosis in breast cancer cells
}

\author{
W. L. SUN*, D. LAN, T. Q. GAN, Z. W. CAI \\ Department of Internal Medicine-Oncology, The First Affiliated Hospital, Guangxi Medical University, Nanning, Guangxi, China, 530021 \\ ${ }^{*}$ Correspondence: swl20022001@hotmail.com
}

Received June 3, 2014 / Accepted August 25, 2014

\begin{abstract}
Acquired multidrug resistance (MDR) is the main mechanism of chemotherapeutic drugs resistance. Nevertheless, the mechanisms of MDR are complex and still not very clear. Recently, including our previous study, several studies have revealed that macroautophagy (here referred to as autophagy) induced by anti-cancer drugs in breast cancer cells may facilitate the development of resistance to epirubicin (EPI), paclitaxel (PTX), tamoxifen or herceptin. Whereas there are a few studies on the relationship between autophagy and MDR, especially the studies designed directly employing induced resistant breast cancer cells. Based on previous study, we explored the relationship between autophagy and MDR. The results showed that induced EPI-resistant MCF-7er and SK-BR-3er cells were simultaneously resistant to PTX and vinorelbine (NVB), which demonstrated that the cells obtained MDR phenotype. Furthermore, PTX and NVB could also induce autophagy in MCF7er and SK-BR-3er cells, and the induced autophagy protected the cells from apoptosis, which facilitated the development of resistance to PTX and NVB. Thus, autophagy promoted the development of MDR in breast cancer cells through inhibition of apoptosis. In addition, we found that P-glycoprotein (Pgp) was overexpressed in MCF-7er and SK-Br-3er cells. And we preliminarily investigated the relationship between autophagy and P-glycoprotein (Pgp). The results showed that the expression of the protein did not obviously change despite the inhibition of autophagy. Therefore, the role of Pgp in the development of MDR might be independent of autophahy. Also this finding implies that autophagy might be a target to overcome MDR in breast cancer cells, and clinical use autophagy inhibitors might be one of the important strategies for overcoming MDR in breast cancer therapy.
\end{abstract}

Key words: autophagy, apoptosis, multidrug resistance, breast cancer, chemotherapy

Epirubicin (EPI), paclitaxel (PTX) and vinorelbine (NVB) are active drugs against breast cancer, and are often used clinically for first- or second-line treatment of breast cancer [1]. Unfortunately, the acquired resistance of breast cancer cells to these drugs seriously limited their use. The mechanisms of resistance are complex and still not entirely clear. It is considered that acquired multidrug resistance (MDR) is the main mechanism of chemotherapeutic drugs resistance [2,3]. However, the molecular mechanisms of MDR are numerous. Among these mechanisms, the one that is the most important and the most studied is ATP-binding cassette $(\mathrm{ABC})$ transport-

Abbreviations: EPI: Epirubicin; PTX: Paclitaxel; NVB: Vinorelbine; MAP1-LC3 or LC3: Microtubule-associated protein1 light chain3; MCF-7er: EPI-resistant MCF-7 cell; MDC: Monodansylcadaverine; MDR: Multidrug resistance ers, which locate on plasma membrane and extrude a broad spectrum of drugs from the cells [4,5,6]. P-glycoprotein (Pgp, also known as $\mathrm{ABCB} 1$ or MDR1), the best studied one of the $A B C$ transporter family, may lead to resistance of anticancer drugs through dramatically decreasing the cellular accumulation of a variety of drugs including EPI, PTX and NVB $[4,5,6]$. It has been proved that the overexpression of Pgp is a main mechanism of MDR [4].

Autophagy is a process of cellular catabolic degradation. It recycles energy from cells own bulk cytoplasm, damaged proteins and organelles, and helps the cell to adapt to starvation or stress (such as hypoxia, $\gamma$-ray and anti-cancer drugs). However, in some cell lines, autophagy also promotes cell death through inducing autophagic cell death (ACD) or enhancing induction of apoptosis, in some cases [7-15]. Recently, a growing amount of evidence suggested that various anticancer therapies (such 
as chemotherapy, hormone therapy, irradiation and targeted therapy) could induce autophagy in diverse of cancer cell lines. Even so, the role of induced autophagy in cancer treatment is still controversial. Some studies showed that autophagy induced by anticancer therapy was a prodeath mechanism, and induction of autophagy was favor for therapy. While more studies showed that induced autophagy was a prosurvival mechanism, which indicated that the induced autophagy might mediate the resistance development of cancer cells for therapy. There have been several comprehensive reviews on it $[8,16,17]$. Our previous study found that the autophagy was significantly increased at basal level as well as after exposing to EPI in induced MCF-7er cells, compared with parental MCF-7 cells. And the induced autophagy protected cells from apoptosis, which facilitated the development of resistance to EPI [18]. The results were lately confirmed again by Chittaranjan et al. in triple negative breast cancer (TNBC) cells [19]. Moreover, several other studies also successively found that induced autophagy contributed to the development of resistance to anticancer drugs (tamoxifen, herceptin or PTX) through inhibiting the occurrence of apoptosis in inducedresistant breast cancer cells [20-22]. However, these studies did not further investigate the function of autophagy in the development of MDR. Consequently, the role of autophagy in MDR has yet to be defined.

In this study, we used parental MCF-7 and SK-BR-3 cells and derived EPI-resistant MCF-7er and SK-BR-3er cells as the cell model to explore the significance of autophagy in the development of MDR. In addition to EPI, we found that MCF7er and SK-BR-3er cells were simultaneous resistance to PTX and NVB. Moreover, PTX and NVB also induced autophagy in MCF-7er and SK-BR-3er cells, and the induced autophagy protected the cells from apoptosis, which contributed MCF-7er and SK-BR-3er cells producing cross-resistance to PTX and NVB. In addition, we found that Pgp, a vital factor of MDR, was overexpressed in MCF-7er and SK-BR-3er cells, and the level of the protein did not obviously change when autophagy was inhibited. Thus, because of upregulating of autophagy, the selection of various doses of EPI confers MDR phenotype on MCF-7er and SK-BR-3er cells. And the upregulated autophagy protects the cells from death through inhibition of apoptosis. Therefore, autophagy has a role in the development of MDR phenotype, which might be independent of Pgp.

\section{Materials and methods}

Agents and antibodies. Monodansylcadaverine (MDC, catalog number: 30432), MTT (catalog number: M2128) and trypan blue (catalog number: T6146) were from Sigma. EPI was from Pfizer Pharmaceuticals Limited (catalog number: H20000496). NVB was from Haosen Pharmaceutical Co. Ltd. (catalog number: 020048). PTX was from Sichuan Taiji Pharmaceutical Co. Ltd. (catalog number: 33069-62-4). ZVAD-fmk (catalog number: KGA8254) was from Nanjing KeyGen Biotech. Co., Ltd.. Rabbit polyclonal anti-human
MAPLC3 $\beta$ (H50) was from Santa Cruz Biotechnology, Inc. (catalog number: sc-28266). Goat polyclonal anti-rabbit $\beta$-actin (catalog number: ab8227), rabbit polyclonal antihuman ATG7 (catalog number: ab53255), rabbit monoclonal anti-human beclin1 (catalog number: ab51031), rabbit polyclonal anti-human active caspase-9 (catalog number: ab2324) and rabbit polyclonal anti-human P-Glycoprotein (catalog number: ab98322) were obtained from Abcam.

Cell culture. MCF-7 cells and SK-BR-3 cells (from American Type Culture Collection) were cultured at $37^{\circ} \mathrm{C}$ in RPMI-1640 supplemented with $10 \%$ fetal calf serum (FCS), 100 units $/ \mathrm{ml}$ penicillin and $100 \mu \mathrm{g} / \mathrm{ml}$ streptomycin in a humid incubator with 5\% $\mathrm{CO}_{2}$. EPI-resistant MCF-7er cells were sustained with $2.5 \mu \mathrm{M}$ EPI, and SK-BR-3er cells with $3.0 \mu \mathrm{M}$ EPI.

EPI-resistant cell (SK-BR-3er cell) induction. Cells were seeded in $25-\mathrm{cm}^{2}$ tissue culture flasks and were allowed to reach about $80 \%$ confluency in fresh medium before treating with EPI. The dose of EPI begun with $0.06 \mu \mathrm{M}$ (1/50 IC50, IC50: $3.0 \mu \mathrm{M}$ in SK-BR-3 cells), and it was increased by a dose gradient that was $25 \sim 50 \%$ of the previous dose. The next dose was given, until the cells were stable in proliferation without significant death. During the induction of SK-BR-3er cells, the cells were subcultured when they reached $\geq 85 \%$ confluency.

Cell proliferation and death assay. For the proliferation assay, cells were seeded at $8 \times 10^{3}$ cells per well in 96-well flatbottomed plates, and were allowed to attach overnight at $37^{\circ} \mathrm{C}$. Afterwards, medium containing agents was added to each well, and cells were further cultured at $37^{\circ} \mathrm{C}$ for the indicated times. The number of viable cells was estimated using MTT. The absorbance was measured at $490 \mathrm{~nm}$ with a microplate reader. The results were from three independent experiments of each group.

For the cell death assay with trypan blue dye exclusion, cells were seeded at $5 \times 10^{5}$ cells in $12.5-\mathrm{cm}^{2}$ tissue culture flasks, and were treated as described for the MTT assay for the indicated times. Cells were trypsinized at the indicated times, and the number of dead cells was counted. Tests were repeated independently three times.

Western blot. For the western blot analysis, cells were seeded in $25-\mathrm{cm}^{2}$ tissue culture flasks and were allowed to reach about $80 \%$ confluency in fresh medium before treatment with the agents. After treatment, detached and attached cells were collected by centrifugation, and whole-cell lysates were obtained using a lysis buffer $(1 \times \mathrm{PBS} \mathrm{pH} 7.6,1 \% \mathrm{NP}-40$, $0.1 \%$ sodium dodecyl sulfate and $0.5 \%$ sodium deoxycholate supplemented with inhibitor cocktails). About 30-50 $\mu \mathrm{g}$ total protein from each group was electrophoretically separated by 12 or $15 \%$ SDS-PAGE, and electrotransferred to polyvinylidene fluoride membranes (PVDF membranes). The PVDF membranes were blocked by $5 \%$ non-fat dry milk in Tris-buffered saline-Tween 20 (TBST, pH 7.6) for $1 \mathrm{~h}$ at room temperature, incubated with light agitation in primary antibodies diluted in $5 \%$ non-fat dry milk in TBST overnight at $4^{\circ} \mathrm{C}$, washed with TBST three times, and incubated with light agitation in secondary antibodies diluted in 5\% non-fat dry milk in TBST for 
$1 \mathrm{~h}$ at room temperature, and then the proteins were detected with electrochemiluminescence (ECL).

Monodansylcadaverine sequestration assay. The MDC sequestration assay was performed as previously described. $[18,20]$ Cells were treated with agents for the indicated time, and then they were treated with MDC $(0.1 \mathrm{mM})$ for $1 \mathrm{~h}$ at $37^{\circ} \mathrm{C}$. Detached and attached cells $\left(\mathrm{n}=2.5 \times 10^{5}\right)$ were collected by centrifugation at 2,500 rpm for $5 \mathrm{~min}$. To the collected cells, $400 \mu \mathrm{l}$ of the lysis buffer was added. The cells were resuspended in the lysis buffer and incubated on ice with light agitation for $30 \mathrm{~min}$. Lysates were centrifuged at 13,000 rpm for $5 \mathrm{~min}$, and then the fluorescence of the supernatant was detected with an emission of $535 \mathrm{~nm}$ and an excitation of $340 \mathrm{~nm}$ with the microplate fluorometer. MDC fluorescence was detected in three independent experiments for each group.

EGFP-LC3 transfection. The pEGFP-LC3 plasmid was a kind gift from Professor T. Yoshimori and Ms. H. Omori (Department of Cellular Regulation Research Institute for Microbial Diseases, Osaka University, Japan). The plasmids were transfected into cells by Lipofectamine ${ }^{\mathrm{TM}} 2000$, the transfection was performed according to the instructions of the manufacturer, and the clones with stable expression of GFP-LC3 were selected by G418.

Fluorescence microscopy. EGFP-tagged LC3 cells $(\mathrm{n}=$ $2 \times 10^{5}$ ) were seeded on coverslips in 6-well plates and attached overnight. Then the cells were treated with agents for the indicated time, and the fluorescence of GFP was assessed with a fluorescence microscope.

Downregulation of BECLIN 1 and ATG7 with short hairpin RNA (shRNA). The human sh-BECLIN 1 targeting sequence was CAGTTTGGCACAATCAATA, and human sh-ATG7 targeting sequence was GGAGTCACAGCTCTTCCTT. [23] The sh-BECLIN 1, ATG7 and control scrambled plasmids were synthesized by GenePharma. The plasmids were transfected into MCF-7er cells by Lipofectamine ${ }^{\mathrm{TM}} 2000$. To assay the downregulation effects on Beclin1 1 and Atg7, the proteins were detected through western blot after transfection with the plasmids for $48 \mathrm{~h}$.

Statistical analyses. Statistical comparisons of mean values were carried out using an Analysis of Variance (ANOVA). $P \leq 0.05$ was considered to be statistically significant.

\section{Results}

MCF-7er and SK-BR-3er cells were cross-resistance to PTX and NVB. As we previously reported, the induced MCF7 er cells could tolerate $6.0 \mu \mathrm{M}$ EPI, and the IC50 of MCF-7er cells to EPI was about four times that of MCF-7 cells $(10.3 \mu \mathrm{M}$ Vs $2.5 \mu \mathrm{M})$.[18] To investigate whether MCF-7er cells were cross-resistance to PTX and NVB, the IC50 of MCF-7 cells and MCF-7er cells to PTX and NVB were tested with MTT. The IC50 of MCF-7 cells to PTX were $8.5 \mathrm{nM}$ and NVB were $1.5 \mu \mathrm{M}$ (Fig. 1A and B). While the IC50 of MCF-7er cells to PTX was about three times that of MCF-7 cells and NVB was about nine times (26.5 nM Vs $8.5 \mathrm{nM}$ and $13.0 \mu \mathrm{M}$ Vs $1.5 \mu \mathrm{M}$, respectively) (Fig. 1A and B). In addition, we did a stepwise selection with various doses of EPI in SK-BR-3 cells to induce EPI resistant cells (SK-BR-3er cells) (Supplementary Fig. S1). SK-BR-3er cells could tolerate $9.0 \mu \mathrm{M}$ EPI, and the IC50 of SK-BR-3er cells $(13.0 \mu \mathrm{M})$ was over four times that of SKBR-3 cells $(3.0 \mu \mathrm{M})$ assayed by MTT (Supplementary Fig. S2). Similarly, the IC50 of SK-BR-3 cells and SK-BR-3er cells to PTX and NVB was tested by MTT. The results showed that the IC50 of SK-BR-3er cells to PTX and NVB was about 2.5 times and 4.0 times that of SK-BR-3 cells $(24.0 \mathrm{nM}$ Vs $9.6 \mathrm{nM}$, $8.5 \mu \mathrm{M}$ Vs $2.0 \mu \mathrm{M}$, respectively) (Fig. 1C and D). Therefore, the induced-EPI resistant MCF-7er and SK-BR-3er cells were cross-resistance to PTX and NVB.

Autophagy was upregulated in MCF-7er and SK-BR-3er cells exposed to PTX and NVB. We had found that MCF-7er cells presented increased level of basal autophagy and increased autophagy in response to EPI treatment when compared to the parental MCF-7 cells.[18] To investigate whether PTX and NVB could also induce autophagy in MCF-7er cells, the cells were respectively treated with $8.5 \mathrm{nM}$ PTX and $1.5 \mu \mathrm{M}$ NVB for $48 \mathrm{~h}$. The cells were then stained with monodansylcadaverine (MDC) and the MDC sequestration assay was tested. MDC is a selective marker for autophagic vacuoles and used to quantitatively detect autophagy.[24] The OD values of the fluorescence of MDC were significantly increased when MCF7 er cells were treated with PTX and NVB (MCF-7er compared with MCF-7er plus PTX, $p<0.001$; MCF-7er compared with MCF-7er plus NVB, $p<0.001$ ) (Fig. 2A). At the same time, the conversion of microtubule-associated protein 1 light chain 3 (MAP1-LC3 or LC3) were detected with western blot. LC3-I is cytosolic and changes to the LC3-II form through conjugating to phosphatidylethanolamine (PE). When autophagy occurs. LC3-II is located on the membrane of phagophores and autophagosomes, and is the only protein in higher eukaryotes that is specifically associated with autophagosomes and a reliable marker of autophagy $[25,26]$. The western blot showed that more LC3-I (18 KDa) was converted into LC3-II (16 KDa) when MCF-7er cells were exposed to PTX and NVB for $48 \mathrm{~h}$ (Fig. 2B). Furthermore, the formation of autophagosomes was observed with the MCF-7er cells expressed of EGFP-tagged LC3 through fluorescence microscopy. In keeping with the increase of MDC fluorescence and LC3-II accumulation in MCF-7er cells, more bright green punctate autophagosomes were observed in EGFP-LC3 MCF-7er cells after exposing to PTX and NVB for $48 \mathrm{~h}$ (Fig. 2C). Also, the OD values of MDC fluorescence remarkably increased when SK-BR-3er cells were treated with PTX, NVB and EPI (SK-Br-3er compared with SK-Br-3er plus PTX, $p=0.001$; SK-Br-3er compared with SK-Br-3er plus NVB, $p<0.001$; SK-Br-3er compared with SKBr-3er plus EPI, $p=0.002$ ). (Fig. 2D). All of the data suggested that autophagy was upregulated in MCF-7er and SK-BR-3er cells exposed to PTX and NVB.

Inhibition of autophagy by sh-BECLIN 1 or sh-ATG7 increased the sensitivity of MCF-7er and SK-BR-3er cells to PTX and NVB. To investigate the function of autophagy 
induced by PTX and NVB in MCF-7er cells, we inhibited autophagy by the down-regulation of Beclin 1 with sh-BECLIN 1. Beclin 1 is one of the indispensible upstream proteins of autophagy in mammal, and it is stable expression in MCF-7 cells. $[18,27]$ After the cells being transfected with sh-BECLIN 1 or scramble sh-RNA for $48 \mathrm{~h}$, the levels of Beclin 1 were detected with western blot. The western blot showed that the level of Beclin 1 significantly decreased in sh-BECLIN 1 group, while the scramble group did not obviously change (Fig. 3A). After treatment with scramble sh-RNA or sh-BECLIN 1 for 48 $\mathrm{h}, \mathrm{MCF}-7 \mathrm{er}$ cells were treated with PTX for another $48 \mathrm{~h}$. Then MDC sequestration assay was tested. The results showed that the OD values of MDC fluorescence of cells treated with sh-
BECLIN 1 plus PTX were significantly lower than that of cells treated with PTX alone or PTX plus scramble sh-RNA (PTX compared with PTX plus sh-BECLIN 1, $p<0.001$; PTX plus scramble compared with PTX plus sh-BECLIN $1, p<0.001$ ). (Fig. 3B). Correspondingly, the formation of autophagosomes in sh-BECLIN 1 EGFP-LC3 MCF-7er cells also dramatically decreased (Fig. 3C). The data indicated that autophagy was inhibited effectively. In accordance with the inhibition of autophagy by sh-BECLIN 1, the viability of MCF-7er cells treated by sh-BECLIN 1 plus PTX also significantly decreased $(p=0.04)$ (Fig. 3D). But the cell death of MCF-7er cells treated with PTX plus sh-BECLIN 1 was still lower than that of parental MCF-7 cells treated with EPI $(p=0.02)$ (Fig. 3D). Meanwhile,
A

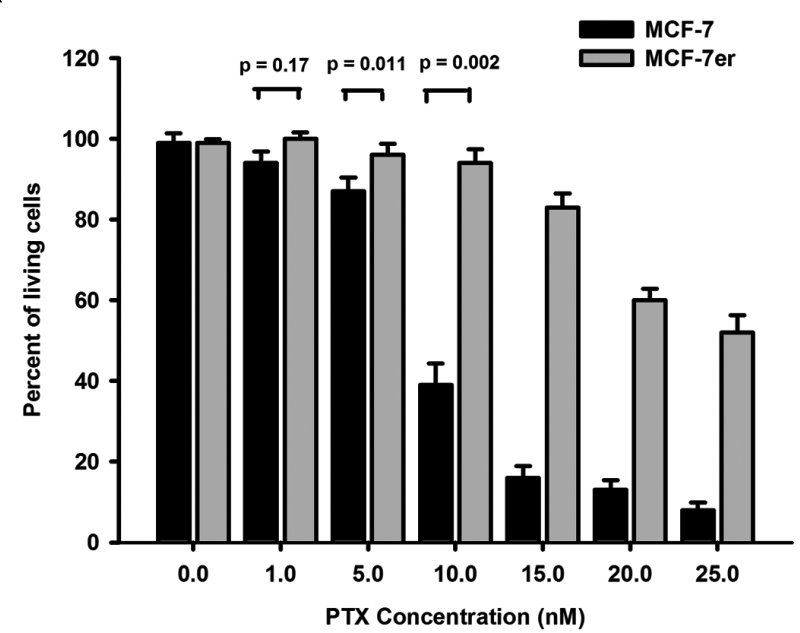

C

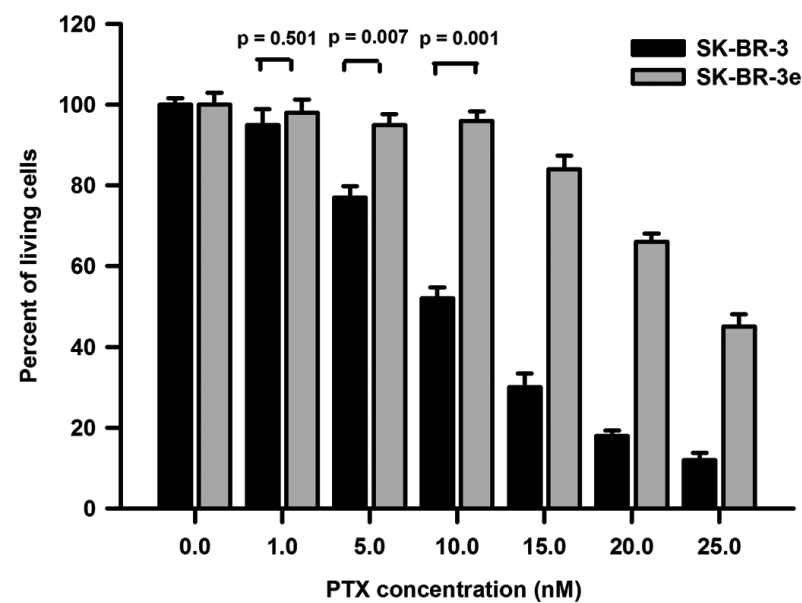

$\mathrm{B}$
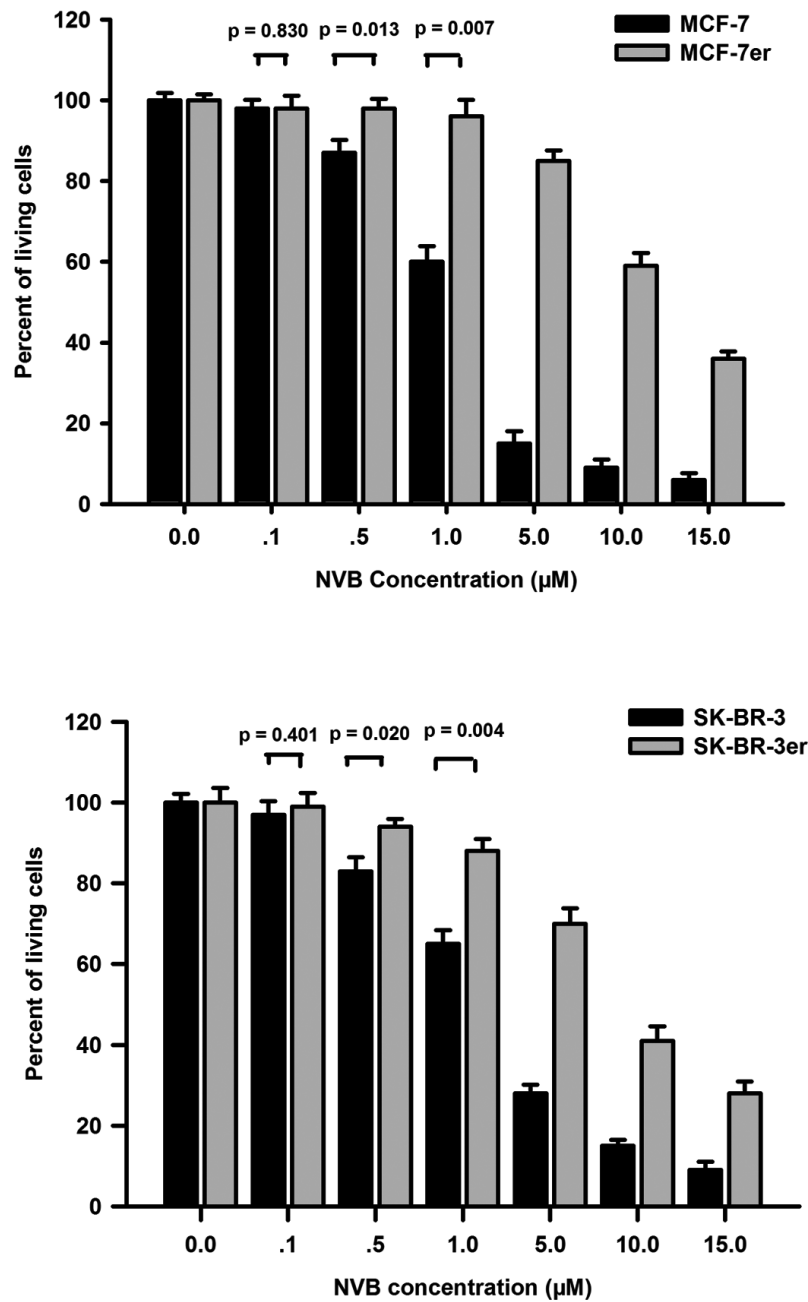

Figure 1. MCF-7er and SK-BR-3er cells were cross-resistance to PTX and NVB. (A) MCF-7 cells and MCF-7er cells were treated with different concentrations of PTX for $48 \mathrm{~h}$, and the proliferation rate was assayed with MTT. The results (mean \pm S.E.) were from three independent experiments. (B) MCF-7 cells and MCF-7er cells were treated with different concentrations of NVB for $48 \mathrm{~h}$, and the proliferation rate was assayed with MTT. The results (mean \pm S.E.) were from three independent experiments. (C) SK-BR-3 cells and SK-BR-3er cells were treated with different concentrations of PTX for 48 h, and the proliferation rate was assayed with MTT. The results (mean \pm S.E.) were from three independent experiments. (D) SK-BR-3 cells and SK-BR-3er cells were treated with different concentrations of NVB for $48 \mathrm{~h}$, and the proliferation rate was assayed with MTT. The results (mean \pm S.E.) were from three independent experiments. 
the cleaved caspase-9 $(37 \mathrm{KDa})$ was detected by western blot. The level of cleaved caspase- 9 was significantly increased when MCF-7er cells were treated by PTX plus sh-BECLIN 1 (Fig. 3E). It indicated that the inhibition of autophagy enhanced apoptosis in MCF-7er cells. And it was confirmed by the increased cell death from autophagy inhibition was inhibited with Z-VADfmk (a pan-caspase inhibitor) (Fig. 3D). Similar results were reached with NVB (data not shown). In addition, to avoid off target effects, we repeated the study with sh-ATG7. Atg7 is a key protein for autophagosomes biogenesis [12]. The level of Atg7 was also dramatically decreased by sh-ATG7, which led to the inhibition of autophagy (Supplementary Fig. S3A and B). And the viability of MCF-7er cells treated by sh-ATG7 plus PTX also significantly decreased ( $p=0.007$ ) (Supplementary Fig. S3C). Similarly, more cleaved caspase- 9 accumulated in MCF-7er cells exposed to PTX plus sh-ATG7, and the increased cell death from autophagy inhibition was inhibited with Z-VADfmk (Supplementary Fig. S3C and D). Also, in SK-BR-3er cells, autophagy was inhibited with sh-BECLIN 1 (Supplementary Fig. S4A and B) and sh-ATG7 (data not shown), which led to increasing of the cell death through induction of apoptosis, when treated with PTX (Supplementary Fig. S4C and D) and NVB (data not shown).

In brief, the autophagy induced by PTX and NVB in MCF7er and SK-BR-3er cells also protected cells from apoptosis. And inhibition of autophagy by sh-BECLIN 1 or sh-ATG7
A

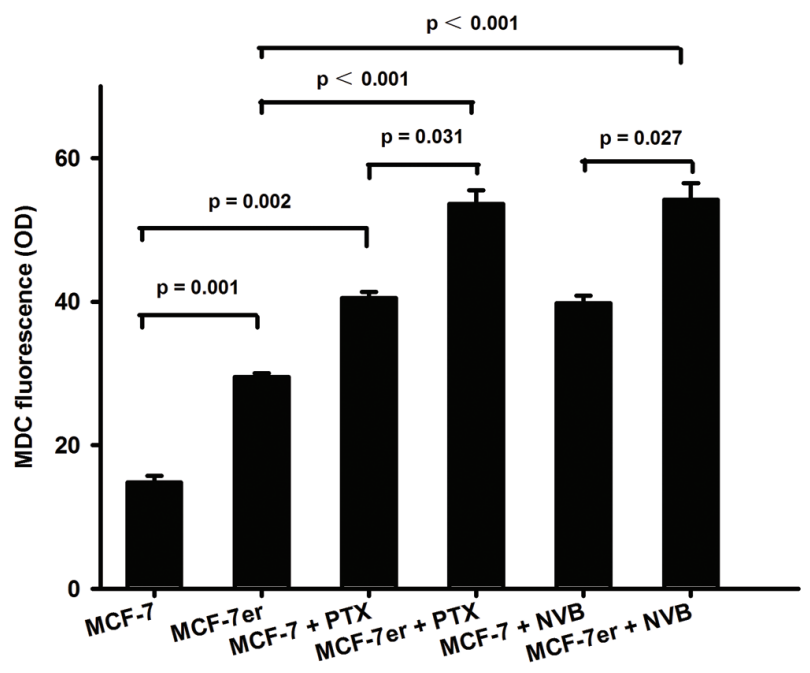

B

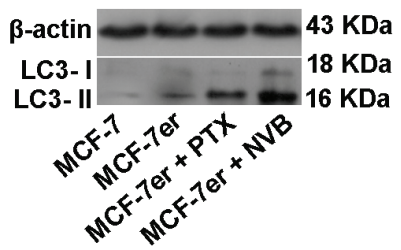

C

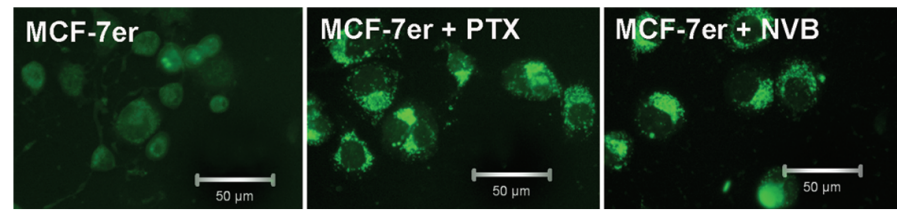

D

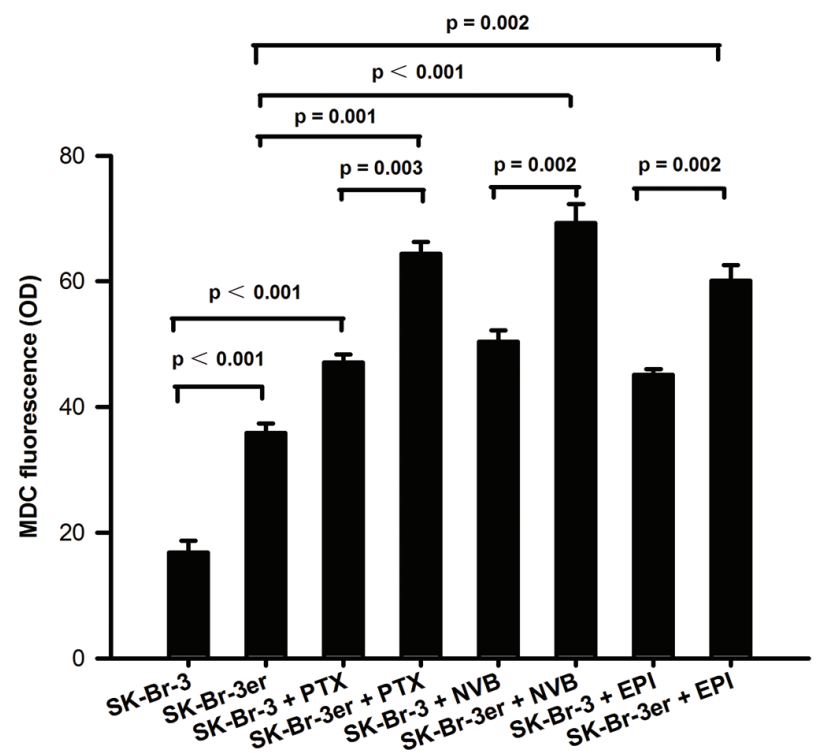

Figure 2. Autophagy was upregulated in MCF-7er and SK-BR-3er cells exposed to PTX and NVB. (A) MCF-7 cells and MCF-7er cells were cultured with or without $8.5 \mathrm{nM}$ PTX or $1.5 \mu \mathrm{M}$ NVB for $48 \mathrm{~h}$, then cells were treated with $0.1 \mathrm{mM}$ monodansylcadaverine (MDC) for $1 \mathrm{~h}$, and MDC sequestration was measured with a microplate fluorometer. The OD values of MDC fluorescence (mean \pm S.E.) were from three independent experiments (MCF-7 compared with MCF-7er, $p=0.001$; MCF-7er compared with MCF-7er plus PTX, $p<0.001$; MCF-7er compared with MCF-7er plus NVB, $p<0.001$ ). (B) LC3-I ( $18 \mathrm{kDa})$ conversion into LC3-II ( $16 \mathrm{kDa})$ was assayed after treatment with or without $8.5 \mathrm{nM}$ PTX or $1.5 \mu \mathrm{M} \mathrm{NVB}$ for $48 \mathrm{~h}$. (C) EGFP-tagged LC3 MCF-7er cells were treated with $8.5 \mathrm{nM}$ PTX or $1.5 \mu \mathrm{M}$ NVB for $48 \mathrm{~h}$; EGFP fluorescence indicates the formation of autophagosomes (200×). (D) SK-BR-3 cells and SK-BR-3er cells were cultured with or without 9.6 nM PTX or $2.0 \mu \mathrm{M} \mathrm{NVB}$ or $3.0 \mu \mathrm{M} \mathrm{EPI} \mathrm{for} 48 \mathrm{~h}$, then cells were treated with $0.1 \mathrm{mM}$ monodansylcadaverine (MDC) for $1 \mathrm{~h}$, and MDC sequestration was measured with a microplate fluorometer. The OD values of MDC fluorescence (mean \pm S.E.) were from three independent experiments (SK-Br-3 compared with SK-Br-3er, $p<0.001$; SK-Br-3er compared with SK-br-3er plus PTX, $p=0.001$; SK-Br-3er compared with SK-br-3er plus NVB, $p<0.001$; SK-Br-3er compared with SK-br-3er plus EPI, $p=0.002$ ). 
increased the sensitivity of MCF-7er and SK-BR-3er cells to PTX and NVB through enhancing induction of apoptosis.

Pgp was overexpressed and independent of autophagy in MCF-7er and SK-BR-3er cells. To investigate the role of ABC family in the resistance development of MCF-7er and SK-BR3er cells to PTX and NVB, basal level of Pgp was detected with western blot in parental MCF-7 and SK-BR-3 cells and derived MCF-7er and SK-BR-3er cells. The results showed that the level of the protein was obviously increased in MCF-7er and SK-BR-3er cells compared with that in parental MCF-7 and SK-BR-3 cells (Fig. 4A). Thus, during the course of induction with various doses of EPI, Pgp was upregulated in MCF-7er and
A

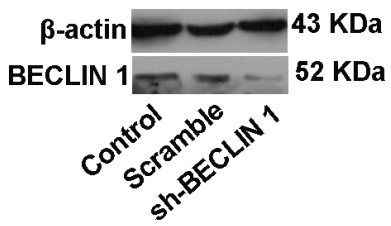

B

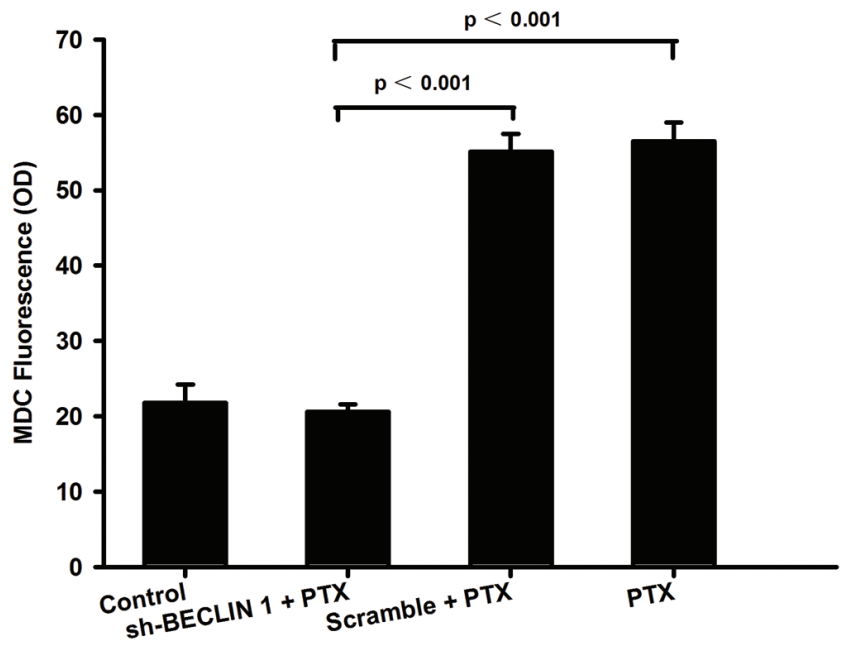

$E$

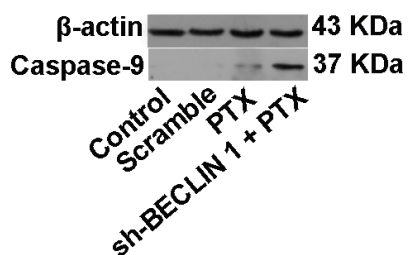

C
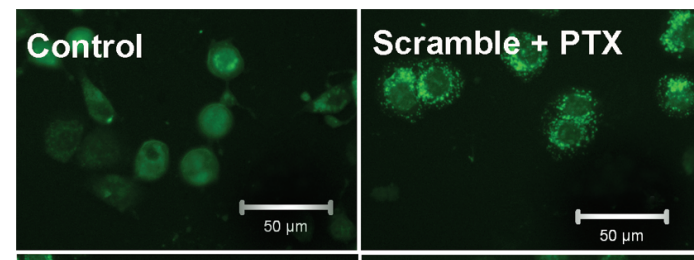

PTX

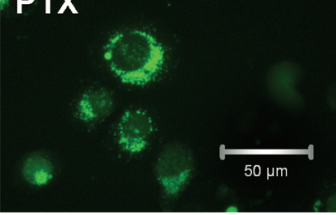

sh-BECLIN $1+$ PTX

$\mathrm{D}$

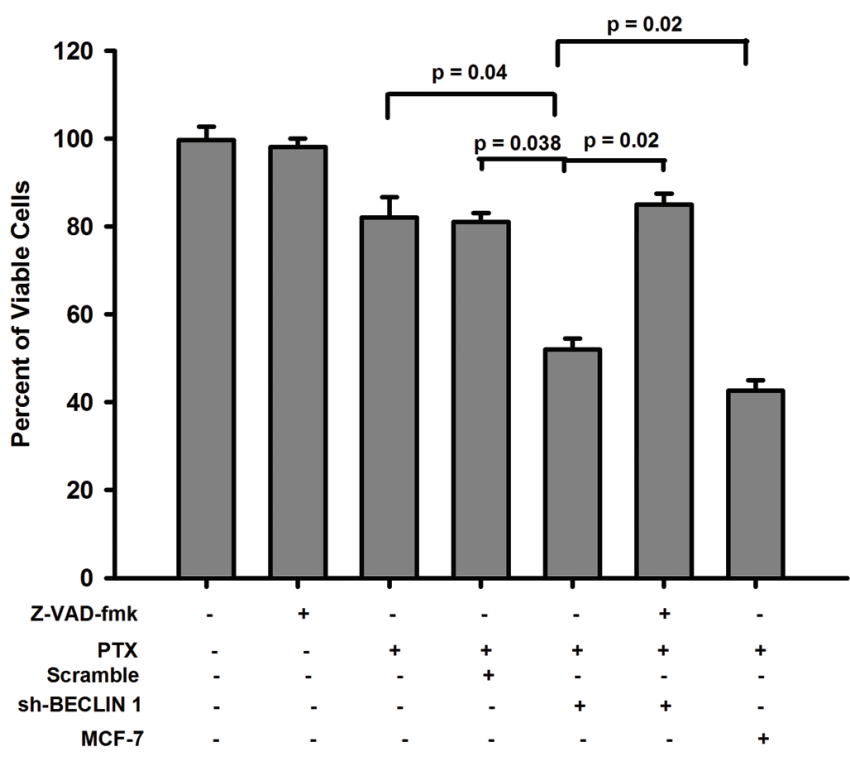

Figure 3. Inhibition of autophagy by sh-BECLIN 1 increased the sensitivity of MCF-7er cells to PTX. (A) Beclin1 was detected with western blot after MCF-7er cells being treated with scramble sh-RNA or sh-BECLIN 1 for $48 \mathrm{~h}$. (B) Cells were treated with scramble sh-RNA or sh-BECLIN 1 for 48 h, followed by treatment with $8.5 \mathrm{nM}$ PTX for another $48 \mathrm{~h}$, then treated with $0.1 \mathrm{mM} \mathrm{MDC} \mathrm{for} 1 \mathrm{~h}$, and MDC sequestration was measured with a microplate fluorometer. The OD values of MDC fluorescence (mean \pm S.E.) were from three independent experiments (PTX compared with PTX plus sh-BECLIN $1, p<0.001$; PTX plus scramble compared with PTX plus sh-BECLIN 1, $p<0.001)$. (C) Cells were treated with scramble sh-RNA or sh-BECLIN 1 for $48 \mathrm{~h}$, followed by treatment with $8.5 \mathrm{nM}$ PTX for another $48 \mathrm{~h}$. EGFP fluorescence indicated autophagosome formation (200×). (D) Cells were treated with scramble sh-RNA or sh-BECLIN 1 for $48 \mathrm{~h}$, followed by treatment with $8.5 \mathrm{nM}$ PTX plus or minus $20 \mu \mathrm{M}$ Z-VAD-fmk for 48 h. Cell viability was measured with trypan blue exclusion. The number of viable cells (mean \pm S.E.) was derived from three independent experiments, and the results are expressed as the relative percentage of each group compared to control (PTX alone compared to PTX plus sh-BECLIN 1, $p=0.04 ;$ PTX plus sh-BECLIN 1 compared with PTX plus sh-BECLIN 1 and Z-VAD-fmk, $p=0.02$; MCF-7 with PTX compared to MCF-7er with PTX plus sh-BECLIN 1, $p=0.02$ ). (E) Cleaved caspase-9 was detected with western blot after MCF-7er cells being treated with scramble sh-RNA or sh-BECLIN 1 for 48 h, followed by treatment with $8.5 \mathrm{nM}$ PTX for $48 \mathrm{~h}$. 
SK-BR-3er cells. Furthermore, to investigate the relationship between autophagy and Pgp in the development of resistance to PTX and NVB, the level of Pgp was detected with western blot when MCF-7er and SK-BR-3er cells were treated with shBECLIN 1 or sh-ATG7 for $48 \mathrm{~h}$. The western blot showed that the level of Pgp did not obviously change when autophagy was inhibited with sh-BECLIN 1 or sh-ATG7 (Fig. $4 \mathrm{~B}$ and C). In addition, the expression of Pgp in MCF-7er and SK-BR-3er cells was not obviously affected by PTX and NVB treatment after autophagy blocking (Fig. 4D). Therefore, the role of Pgp might be independent of autophagy in the resistance development of MCF-7er and SK-BR-3er cells to PTX and NVB.

\section{Discussion}

In this study, we showed that induced EPI-resistant MCF7er and SK-BR-3er cells were cross-resistance to PTX and NVB. Moreover, PTX and NVB could induce autophagy in MCF-7er and SK-BR-3er cells. And the induced autophagy facilitated the development of acquired MDR phenotype through inhibition of apoptosis.

Recently, a series of studies demonstrated that autophagy induced by anti-cancer treatment was a protective mechanism, which indicated that autophagy might mediate the resistance of cancer cells to anticancer treatment [28-49]. As a matter of fact, we and other several studies had confirmed the inference using induced resistant breast cancer cell model [18-22]. However, these studies did not directly investigate the relationship between autophagy and MDR, which is the main mechanism of anticancer drugs resistance. Similar to EPI, we found that the IC50 of MCF-7er and SK-BR-3er cells to PTX and NVB was significantly increased compared to that of parental MCF-7 and SK-BR-3 cells. So MCF-7er and SK-BR-3er cells presented resistance to EPI, PTX and NVB simultaneously. The phenomenon was in agreement with the concept of MDR. The relationship between autophagy and apoptosis is complex, they are mutual inhibition, under some conditions $[15,50]$. In our previous study, mitochondria was damaged in a short time when MCF-7 cells were treated with EPI, but only a few of cells exhibited apoptosis, and more cells underwent autophagy. The reasons lay in autophagosomes engulfed the damaged mitochondria, which led to insufficient cytochrome c being released to activate caspase- 9 and apoptosis [18]. In this study, there was only a small amount of cleaved caspase- 9 accumulation in MCF-7er and SK-BR-3er cells when treated with PTX and NVB, and cells presented more autophagy. However, the level of cleaved caspase- 9 dramatically increased when autophagy was inhibited by sh-BECLIN 1 or sh-ATG7, and the cell death of MCF-7er and SK-BR-3er cells was also dramatically increased accordingly. So, autophagy induced by PTX and NVB protected MCF-7er cells from cell death through inhibiting the occurrence of apoptosis, too. It was confirmed again by the decrease of increased cell death due to inhibition of autophagy after inhibition of apoptosis with Z-VAD-fmk. Therefore, autophagy played a role in the development of MDR phenotype in MCF-7er and SK-BR-3er cells
A

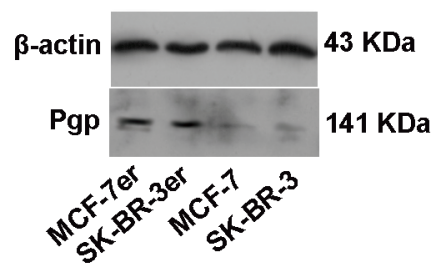

C

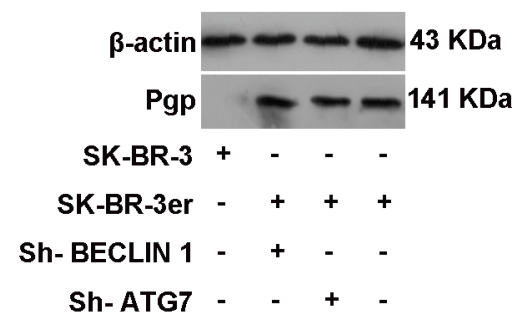

B

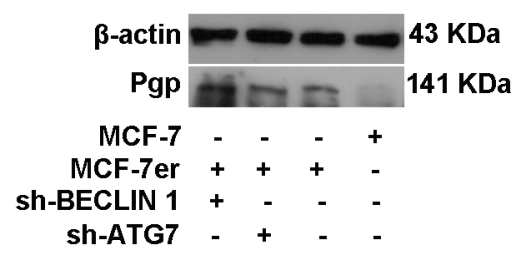

D

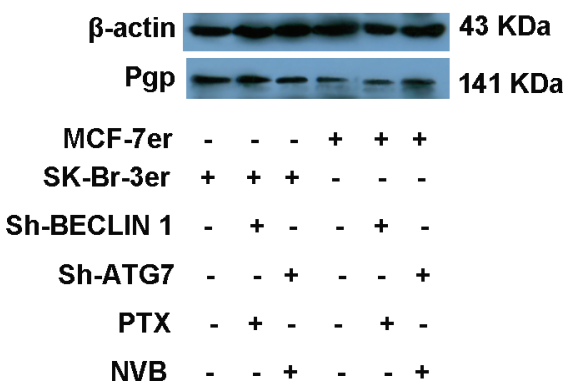

Figure 4. Pgp was overexpressed and independent of autophagy in MCF-7er and SK-BR-3er cells. (A) The basal level of Pgp was detected with western blot in MCF-7 cells, SK-BR-3 cells, MCF-7er and SK-BR-3er cells. (B) Pgp was detected with western blot in MCF-7 and MCF-7er cells after treatment with sh-BECLIN 1 or sh-ATG7 for 48 h. (C) Pgp was detected with western blot in SK-BR-3 and SK-BR-3er cells after treatment with sh-BECLIN 1 or sh-ATG7 for 48 h. (D) Pgp was detected with western blot in SK-BR-3 and SK-BR-3er cells after treatment with sh-BECLIN 1 or sh-ATG7 for $48 \mathrm{~h}$, then treatment with PTX or NVB for another $48 \mathrm{~h}$. 
through inhibition of apoptosis. It was worthwhile to note that the cell death of MCF-7er and SK-BR-3er cells treated with shBECLIN 1 or sh-ATG7 plus PTX or NVB was still lower than that of parental MCF-7 and SK-BR-3 cells treated with PTX or NVB. It further illustrates the complexity of the mechanisms of MDR, and autophagy is just one of them.

It is well known that the molecular mechanisms of MDR are intricate, and there are a number of factors involved in, including decreased uptake of drugs, decreased capacity of drugs to getting rid of cells owing to various changes in cells, increased efflux pump and so on [4]. ABC transporters are the most important and the most studied efflux pumps. And Pgp is the best studied one of the ABC transporter families, which is mainly exhibited in the transport epithelium of the liver, kidney and gastrointestinal tract, at pharmacological barrier sites.[5] The protein may discharge a large variety of chemotherapeutic drugs from the cell, including EPI, PTX and NVB, leading to insufficient drugs accumulation to kill the cells. It has been found that Pgp is wildly expressed in many human cancers, which is related to poor clinical outcome of breast cancer, sarcoma and some types of leukemia [5]. Thus the overexpression of Pgp is a pivotal factor of the MDR phenotype development. Recently, Ajabnoor et al. found that the induced paclitaxel-resistant MCF-7TaxR cells overexpressed Pgp, but the cells did not show obvious cross-resistance to other anticancer drugs (doxorubicin, etoposide, cisplatin and carboplatin) [22]. While, our results showed that the MCF-7er and SK-BR-3er cells overexpressed Pgp and were simultaneous resistance to different chemotherapeutic drugs EPI, PTX and NVB. Therefore, the protein of Pgp also played a role in the development of acquired MDR phenotype in MCF-7er and SK-BR-3er cells.

As mentioned above, Pgp and autophagy were involved in the development of MDR in MCF-7er and SK-BR-3er cells simultaneously. Following, we preliminarily investigated the relationship between them. And the results showed that the sensitivity of MCF-7er and SK-BR-3er cells to PTX and NVB was restored after inhibition of autophagy, while the level of Pgp did not obviously change. Therefore, the role of the protein might be independent of autophagy in the development of MDR. But it needs further study.

In brief, our study showed that the induced MCF-7er and SK-BR-3er cells obtained MDR phenotype. Similar to EPI, PTX and NVB could also induce autophagy in MCF-7er and SK-BR-3er cells. Moreover, the induced autophagy protected the cells from apoptosis, which facilitated the development of resistance to these drugs. Therefore, autophagy played a role in the development of MDR phenotype. At the same time, MCF-7er and SK-BR-3er cells overexpressed Pgp, which was independent of autophagy in MDR development. Our results suggested that autophagy might be a target to overcome MDR in breast cancer therapy. Nevertheless, the molecular mechanisms of autophagy and acquired MDR, and the relationship between autophagy and other factors of acquired MDR need further studies.

\section{Conclusions}

Above of all, we conclude that autophagy facilitates the development of MDR in breast cancer cells through inhibition of apoptosis. And it might be independent of Pgp in MDR. Moreover, this finding indicates that autophagy might be a target to overcome MDR, and the combination of chemotherapy and an autophagy inhibitor might be a potent therapeutic strategy for overcoming MDR in breast cancer treatment.

Supplementary information is available in the online version of the paper.

Acknowledgements: This study is supported by grants from the National Natural Science Foundation of China (grant number 81360340) and Wu Jieping Medical Foundation clinical research special fund (grant number 320.6750.12689). We thank Professor T. Yoshimori and Ms. H. Omori (Department of Cellular Regulation Research Institute for Microbial Diseases, Osaka University, Japan) for kindly providing the pEGFP-LC3 plasmid.

\section{References}

[1] NCCN Clinical Practice Guidelines in Oncology (NCCN Guidelines) ${ }^{\circledR}$, Breast Cancer Version 2.2014. http://www.nccn. org.

[2] GOTTESMAN MM. Mechanisms of cancer drug resistance. Annu Rev Med 2002; 53: 615-627. http://dx.doi.org/10.1146/ annurev.med.53.082901.103929

[3] NIELSEN D, MAARE C, SKOVSGAARD T. Cellular resistance to anthracyclines. Gen Pharmacol 1996; 27: 251-255. http://dx.doi.org/10.1016/0306-3623(95)02013-6

[4] SZAKACS G, PATERSON JK, LUDWIG JA, BOOTH-GENTHE C, GOTTESMAN MM. Targeting multidrug resistance in cancer. Nature Rev Drug Discovery, 2006; 5: 219-234. http://dx.doi.org/10.1038/nrd1984

[5] SCHINKEL AH, JONKER JW. Mammalian drug efflux transporters of the ATP binding cassette (ABC) family: an overview. Advanced Drug Delivery Rev, 2012; 64: 138-153. http://dx.doi.org/10.1016/j.addr.2012.09.027

[6] AMBUDKAR SV, DEY S, HRYCYNA CA, RAMACHANDRA M, PASTAN I et al. Biochemical, cellular, and pharmacological aspects of the multidrug transporter. Annu Rev Pharmacol Toxicol, 1999; 39: 361-398. http://dx.doi.org/10.1146/annurev. pharmtox.39.1.361

[7] KROEMER G, JAATTELA M. Lysosomes and autophagy in cell death control. Nature Rev Cancer 2005; 5: 886-897. http:// dx.doi.org/10.1038/nrc1738

[8] KONDO Y, KANZAWA T, SAWAYA R, KONDO S. The role of autophagy in cancer development and response to therapy. Nature Rev Cancer 2005; 5: 726-734. http://dx.doi. org/10.1038/nrc1692

[9] XIE ZP, KLIONSKY DJ. Autophagosome formation: core machinery and adaptation. Nature Cell Biol 2007; 9: 1102-1109. http://dx.doi.org/10.1038/ncb1007-1102 
[10] KLIONSKY DJ. Autophagy: from phenomenology to molecular understanding in less than a decade. Nature Rev Mol Cell Biol 2007; 8: 931-937. http://dx.doi.org/10.1038/ nrm2245

[11] LEVINE B. Autophagy and cancer. Nature 2007; 446: 745-747. http://dx.doi.org/10.1038/446745a

[12] RAVIKUMAR B, FUTTER M, JAHREISS L, KOROLCHUK VI, LICHTENBERG $\mathrm{M}$ et al. Mammalian macroautophagy at a glance. J Cell Sci 2009; 122: 1707-1711. http://dx.doi. org/10.1242/jcs.031773

[13] BAEHRECKE EH. Autophagy: dual roles in life and death? Nature Rev Mol Cell Biol 2005; 6: 505-510. http://dx.doi. org/10.1038/nrm1666

[14] LEVINE B, YUAN J. Autophagy in cell death: an innocent convict? J Clin Invest 2005; 115: 2679-2688. http://dx.doi. org/10.1172/JCI26390

[15] MAIURI MC, ZALCKVAR E, KIMCHI A, KROEMER G. Self-eating and self-killing: crosstalk between autophagy and apoptosis. Nature Rev Mol Cell Biol 2007; 8: 741-752. http:// dx.doi.org/10.1038/nrm2239

[16] SUI X, CHEN R, WANG Z, HUANG Z, KONG N et al.. Autophagy and chemotherapy resistance: a promising therapeutic target for cancer treatment. Cell Death and Disease 2013; 4, e838. http://dx.doi.org/10.1038/cddis.2013.350

[17] LORINA S, HAMAÏB A, MEHRPOURB M, CODOGNOB P. Autophagy regulation and its role in cancer. Seminars in Cancer Biology 2013; 23: 361- 379. http://dx.doi.org/10.1016/j. semcancer.2013.06.007

[18] SUN WL, CHEN J, WANG YP, ZHENG H. Autophagy protects breast cancer cells from epirubicin-induced apoptosis and facilitates epirubicin-resistance development. Autophagy 2011; 7: 1035-1044. http://dx.doi.org/10.4161/ auto.7.9.16521

[19] CHITTARANJAN S, BORTNIK S, DRAGOWSKA WH, XU J, ABEYSUNDARA N et al.. Autophagy Inhibition Augments the Anticancer effects of Epirubicin treatment in Anthracycline-Sensitive and Resistant Triple Negative Breast Cancer. Clin Cancer Res 2014; Published Online First.

[20] SAMADDAR JS, GADDY VT, DUPLANTIER J, THANDAVAN SP, SHAH M et al. A role for macroautophagy in protection against 4-hydroxytamoxifen-induced cell death and the development of antiestrogen resistance. Mol Cancer Ther 2008; 7: 2977-2987. http://dx.doi.org/10.1158/15357163.MCT-08-0447

[21] VAZQUEZ-MARTIN A, OLIVERAS-FERRAROS C, MENENDEZ JA. Autophagy Facilitates the Development of Breast Cancer Resistance to the Anti-HER2 Monoclonal Antibody Trastuzumab. PLoS ONE, 2009; 4, e6251. http:// dx.doi.org/10.1371/journal.pone.0006251

[22] AJABNOOR GMA, CROOK T, COLEY HM. Paclitaxel resistance is associated with switch from apoptotic to autophagic cell death in MCF-7 breast cancer cells. Cell Death and Disease 2012; 3: e260. http://dx.doi.org/10.1038/cddis.2011.139

[23] YU L, ALVA A, SU H, DUTT P, FREUNDT E et al. Regulation of an ATG7-beclin 1 program of autophagic cell death by caspase-8. Science 2004; 304: 1500-1502. http://dx.doi. org $/ 10.1126 /$ science. 1096645
[24] BIEDERBICK A, KERN HF, ELSASSER HP. Monodansylcadaverine (MDC) is a specific in vivo marker for autophagic vacuoles. Eur J Cell Biol 1995; 66: 3-14.

[25] KABEYA Y, MIZUSHIMA N, UENO T, YAMAMOTO A, KIRISAKO T et al. LC3, a mammalian homologue of yeast Apg8p, is localized in autophagosome membranes after processing. EMBO J. 2000; 19: 5720-5728. http://dx.doi. org/10.1093/emboj/19.21.5720

[26] KLIONSKY DJ, ABELIOVICH H, AGOSTINIS P, AGRAWAL DK, ALIEV G et al. Guidelines for the use and interpretation of assays for monitoring autophagy in higher eukaryotes. Autophagy 2008; 4: 151-175. http://dx.doi.org/10.4161/auto.5338

[27] LIANG XH, JACKSON S, SEAMAN M, BROWN K, KEMPKES B et al. Induction of autophagy and inhibition of tumorigenesis by beclin 1 . Nature 1999; 402: 672-676. http:// dx.doi.org/10.1038/45257

[28] LI J, HOU N, FARIED A, TSUTSUMI S, KUWANO H. Inhibition of autophagy augments 5-fluorouracil chemotherapy in human colon cancer in vitro and in vivo model. Eur J Cancer 2010; 46: 1900-1909. http://dx.doi.org/10.1016/j. ejca.2010.02.021

[29] DE LA CRUZ-MORCILLO MA, VALERO ML, CALLEJASVALERA JL, ARIAS-GONZA'LEZ L, MELGAR-ROJAS P et al. P38MAPK is a major determinant of the balance between apoptosis and autophagy triggered by 5-fluorouracil: implication in resistance. Oncogene 2012; 31: 1073-1085. http:// dx.doi.org/10.1038/onc.2011.321

[30] SASAKI K, TSUNO NH, SUNAMI E, TSURITA G, KAWAI $\mathrm{K}$ et al. Chloroquine potentiates the anti-cancer effect of 5-fluorouracil on colon cancer cells. BMC Cancer 2010; 10: 370. http://dx.doi.org/10.1186/1471-2407-10-370

[31] SASAKI K, TSUNO NH, SUNAMI E, KAWAI K, HONGO $\mathrm{K}$ et al. Resistance of colon cancer to 5-fluorouracil may be overcome by combination with chloroquine, an in vivo study. Anticancer Drugs 2012; 23: 675-682. http://dx.doi. org/10.1097/CAD.0b013e328353f8c7

[32] YANG PM, LIU YL, LIN YC, SHUN CT, WU MS et al. Inhibition of autophagy enhances anticancer effects of atorvastatin in digestive malignancies. Cancer Res 2010; 70: 7699-7709. http://dx.doi.org/10.1158/0008-5472.CAN-10-1626

[33] PAILlAS S, CAUSSE A, MARZI L, DE MEDINA P, POIROT $M$ et al. MAPK14/p38a confers irinotecan resistance to TP53-defective cells by inducing survival autophagy. Autophagy 2012; 8: 1098-1112. http://dx.doi.org/10.4161/ auto. 20268

[34] LIU D, YANG Y, LIU Q, WANG J. Inhibition of autophagy by 3-MA potentiates cisplatin induced apoptosis in esophageal squamous cell carcinoma cells. Med Oncol 2011; 28: 105-111. http://dx.doi.org/10.1007/s12032-009-9397-3

[35] DING ZB, HUI B, SHI YH, ZHOU J, PENG YF et al. Autophagy activation in hepatocellular carcinoma contributes to the tolerance of oxaliplatin via reactive oxygen species modulation. Clin Cancer Res 2011; 17: 6229-6238. http:// dx.doi.org/10.1158/1078-0432.CCR-11-0816

[36] GUO XL, LI D, SUN K, WANG J, LIU Y et al. Inhibition of autophagy enhances anticancer effects of bevacizumab in 
hepatocarcinoma. J Mol Med (Berl) 2013; 91: 473-483. http:// dx.doi.org/10.1007/s00109-012-0966-0

[37] SHI YH, DING ZB, ZHOU J, HUI B, SHI GM et al. Targeting autophagy enhances sorafenib lethality for hepatocellular carcinoma via ER stress-related apoptosis. Autophagy 2011; 7: 1159-1172. http://dx.doi.org/10.4161/auto.7.10.16818

[38] ZHAO M, YANG M, YANG L, YU Y, XIE M et al. HMGB1 regulates autophagy through increasing transcriptional activities of JNK and ERK in human myeloid leukemia cells. BMB Rep 2011; 44: 601-606. http://dx.doi.org/10.5483/ BMBRep.2011.44.9.601

[39] Han W, Pan H, Chen Y, Sun J, Wang Y et al. EGFR tyrosine kinase inhibitors activate autophagy as a cytoprotective response in human lung cancer cells. PLoS One 2011; 6: e18691. http://dx.doi.org/10.1371/journal.pone.0018691

[40] WANG Y, PENG RQ, LI DD, DING Y, WU XQ et al. Chloroquine enhances the cytotoxicity of topotecan by inhibiting autophagy in lung cancer cells. Chin J Cancer 2011; 30: 690-700. http://dx.doi.org/10.5732/cjc.011.10056

[41] KANG R, TANG D, SCHAPIRO NE, LIVESEY KM, FARKAS A et al. The receptor for advanced glycation end products (RAGE) sustains autophagy and limits apoptosis, promoting pancreatic tumor cell survival. Cell Death Differ 2010; 17: 666-676. http://dx.doi.org/10.1038/cdd.2009.149

[42] SHIN SW, KIM SY, PARK JW. Autophagy inhibition enhances ursolic acid-induced apoptosis in PC3 cells. Biochim Biophys Acta 2012; 1823: 451-457. http://dx.doi.org/10.1016/j. bbamcr.2011.10.014

[43] SHINGU T, FUJIWARA K, BO“ GLER O, AKIYAMA Y, MORITAKE $\mathrm{K}$ et al. Inhibition of autophagy at a late stage enhances imatinib-induced cytotoxicity in human malignant glioma cells. Int J Cancer 2009; 124: 1060-1071. http://dx.doi. org/10.1002/ijc.24030
[44] SCHOENLEIN PV, PERIYASAMY-THANDAVAN S, SAMADDAR JS, JACKSON WH, BARRETT JT. Autophagy facilitates the progression of ERalpha-positive breast cancer cells to antiestrogen resistance. Autophagy 2009; 5: 400-403. http://dx.doi.org/10.4161/auto.5.3.7784

[45] O'DONOVAN TR, O'SULLIVAN GC, MCKENNA SL. Induction of autophagy by drug-resistant esophageal cancer cells promotes their survival and recovery following treatment with chemotherapeutics. Autophagy 2011; 7: 509-524. http:// dx.doi.org/10.4161/auto.7.5.15066

[46] CHEN YS, SONG HX, LU Y, LI X, CHEN T et al. Autophagy inhibition contributes to radiation sensitization of esophageal squamous carcinoma cells. Dis Esophagus 2011; 24: 437-443. http://dx.doi.org/10.1111/j.1442-2050.2010.01156.x

[47] XU CX, ZHAO L, YUE P, FANG G, TAO H et al. Augmentation of NVP-BEZ235's anticancer activity against human lung cancer cells by blockage of autophagy. Cancer Biol Ther 2011; 12: 549-555. http://dx.doi.org/10.4161/ cbt.12.6.16397

[48] MIRZOEVA OK, HANN B, HOM YK, DEBNATH J, AFTAB $\mathrm{D}$ et al. Autophagy suppression promotes apoptotic cell death in response to inhibition of the PI3K-mTOR pathway in pancreatic adenocarcinoma. J Mol Med (Berl) 2011; 89: 877-889. http://dx.doi.org/10.1007/s00109-011-0774-y

[49] XU WH, LIU ZB, HOU YF, HONG Q, HU DL et al. Inhibition of autophagy enhances the cytotoxic effect of PA-MSHA in breast cancer. BMC Cancer 2014; 14: 273. http://dx.doi. org/10.1186/1471-2407-14-273

[50] BINCOLETTO C, BECHARA A, PEREIRA JGS, SANTOS $\mathrm{CP}, \mathrm{ANTUNES} \mathrm{F}$ et al. Interplay between apoptosis and autophagy, a challenging puzzle: New perspectives on antitumor chemotherapies. Chemico-Biological Interactions 2013; 206 : 279-288. http://dx.doi.org/10.1016/j.cbi.2013.09.018 


\title{
Supplementary Information
}

\section{Autophagy facilitates multidrug resistance development through inhibition of apoptosis in breast cancer cells}

\author{
W. L. SUN*, D. LAN, T. Q. GAN, Z. W. CAI
}

Department of Internal Medicine-Oncology, The First Affiliated Hospital, Guangxi Medical University, Nanning, Guangxi, China, 530021

*Correspondence: swl20022001@hotmail.com

Supplementary Figure

SK-BR-3
$\downarrow$
0.06
$\downarrow$
0.075
$\downarrow$
0.1
$\downarrow$
0.125
$\downarrow$
0.15
$\downarrow$
0.225
$\downarrow$
0.35
$\downarrow$
0.525
$\downarrow$
0.8
$\downarrow$
1.2
$\downarrow$
1.8
$\downarrow$
2.7
$\downarrow$
4.0
$\downarrow$
6.0
$\downarrow$
7.5
$\downarrow$
9.0
$\downarrow$
SK-BR-3er

Figure S1. Stepwise drug selection to establish EPI-resistant SK-BR-3 cells (SK-BR-3er cells). The dose of EPI began with $0.06 \mu \mathrm{M}$ (1/50 IC50, IC50: $3.0 \mu \mathrm{M}$ in SK-BR-3 cells), and it was increased by a dose gradient that was $25 \sim 50 \%$ of the previous dose. SK-BR-3er cells could tolerate $9.0 \mu \mathrm{M}$ EPI. During the stepwise drug selection process, cells were not clonal selected.

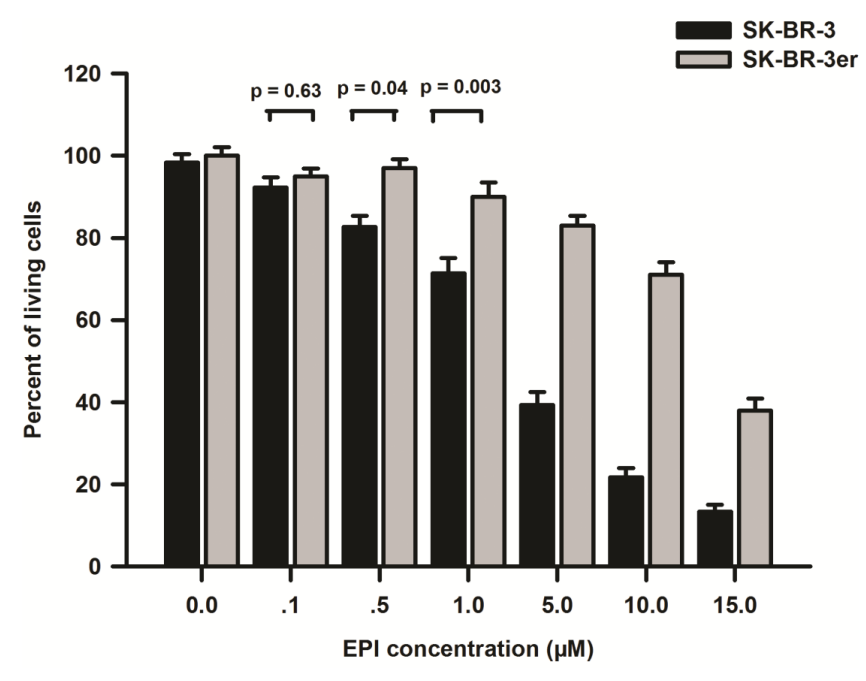

Figure S2. IC50 of SK-BR-3 cells and SK-BR-3er cells to EPI. SK-BR-3 cells and SK-BR-3er cells were treated with a different concentrations of EPI for $48 \mathrm{~h}$, and the proliferation rate was assayed with MTT. The results (mean \pm S.E.) were from three independent experiments. 
A

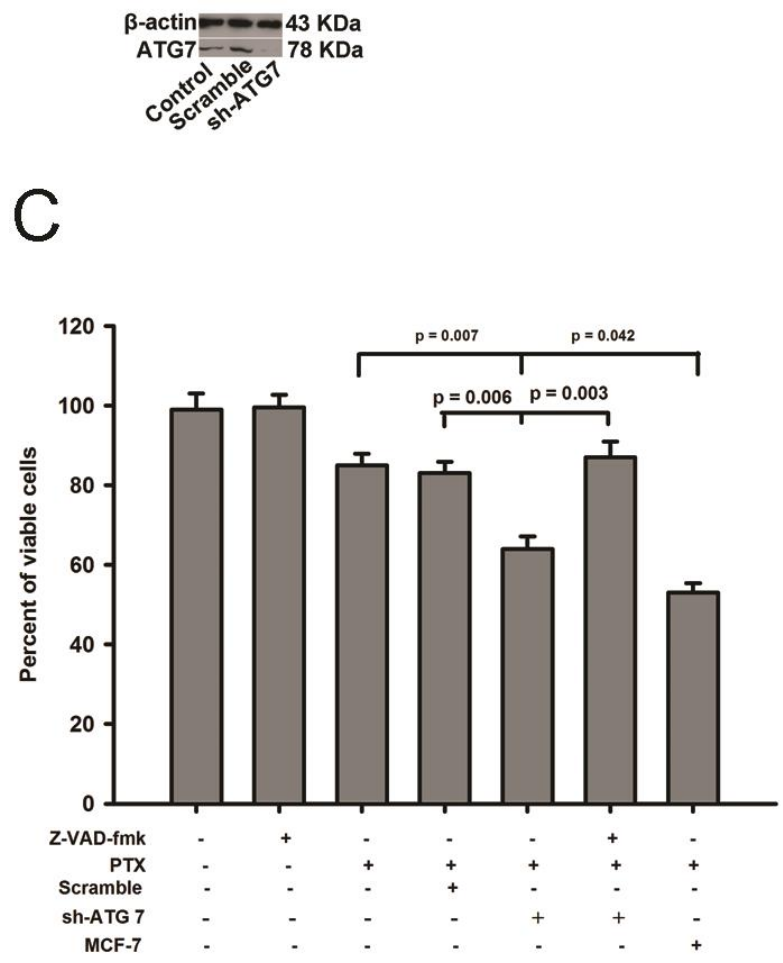

B

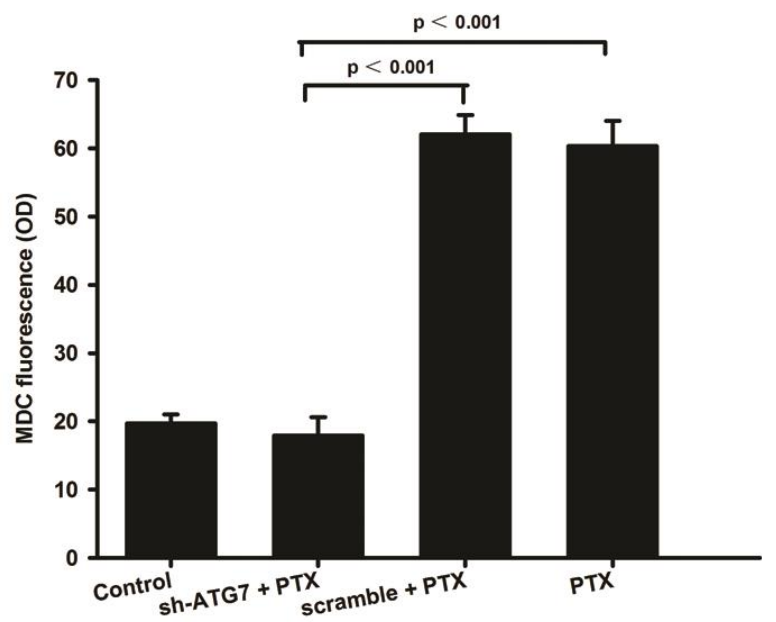

D

Figure S3. Inhibition of autophagy by sh-ATG7 increased the sensitivity of MCF-7er cells to PTX. (A) Atg7 was detected with western blot after MCF-7er cells being treated with scramble sh-RNA or sh-ATG7 for $48 \mathrm{~h}$. (B) Cells were treated with scramble sh-RNA or sh-ATG7 for 48 h, followed by treatment with $8.5 \mathrm{nM}$ PTX for another $48 \mathrm{~h}$, then treated with $0.1 \mathrm{mM}$ MDC for $1 \mathrm{~h}$, and MDC sequestration was measured with a microplate fluorometer. The OD values of MDC fluorescence (mean \pm S.E.) were from three independent experiments (PTX compared with PTX plus sh-ATG7, $p<0.001$; PTX plus scramble compared with PTX plus sh-ATG7, $p<0.001)$. (C) Cells were treated with scramble sh-RNA or sh-ATG7 for $48 \mathrm{~h}$, followed by treatment with $8.5 \mathrm{nM}$ PTX plus or minus $20 \mu \mathrm{M}$ Z-VAD-fmk for $48 \mathrm{~h}$. Cell viability was measured with trypan blue exclusion. The number of viable cells (mean \pm S.E.) was derived from three independent experiments, and the results are expressed as the relative percentage of each group compared to control (PTX alone compared to PTX plus sh-ATG7, P = 0.007; PTX plus sh-ATG7 compared with PTX plus sh-ATG7 and Z-VAD-fmk, $p=0.003$; MCF-7 with PTX compared to MCF-7er with PTX plus sh-ATG7, $p=0.042$ ). (D) Cleaved caspase-9 was detected with western blot after MCF-7er cells being treated with scramble sh-RNA or sh-ATG7 for $48 \mathrm{~h}$, followed by treatment with $8.5 \mathrm{nM}$ PTX for $48 \mathrm{~h}$. 

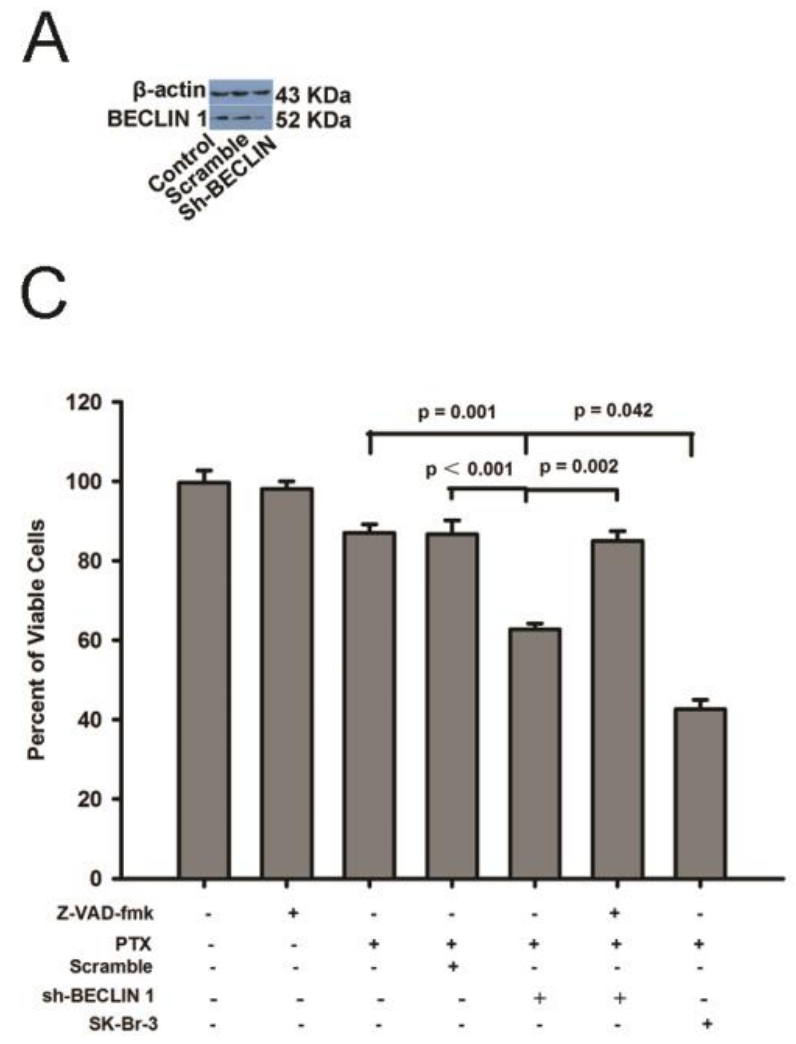
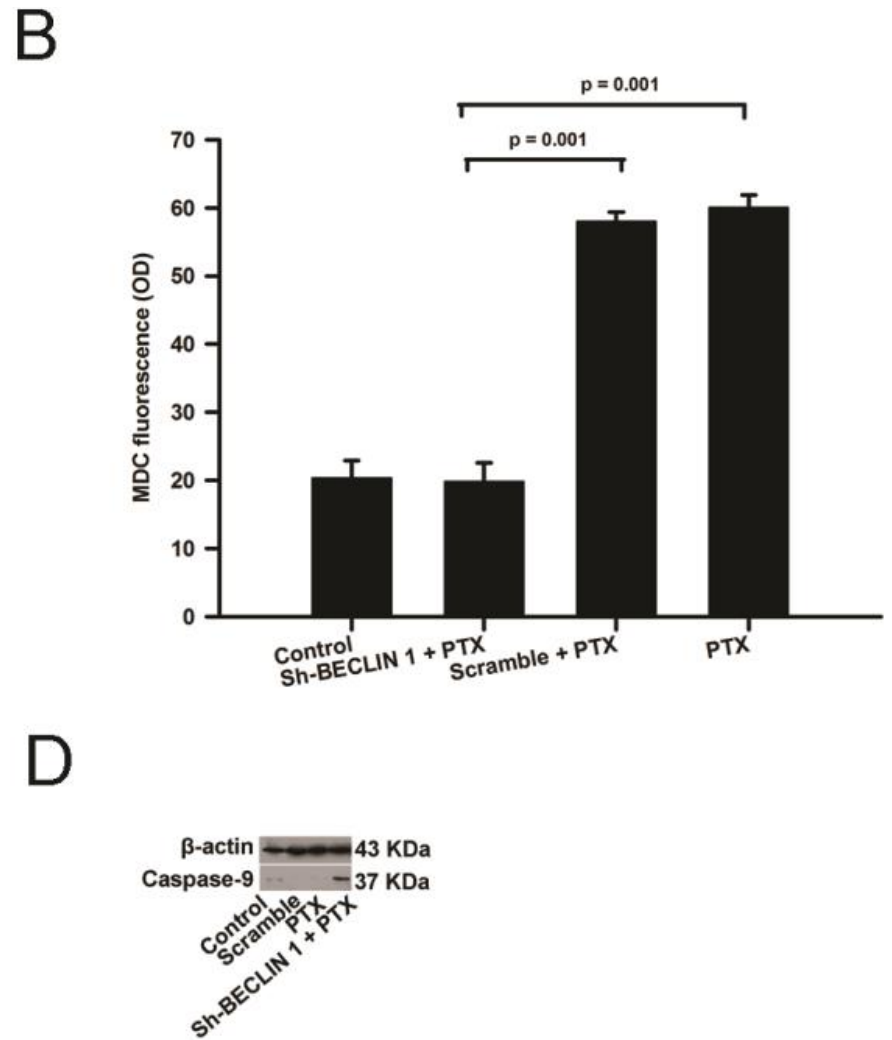

Figure S4. Inhibition of autophagy by sh-BECLIN 1 increased the sensitivity of SK-BR-3er cells to PTX. (A) Beclin 1 was detected with western blot after SK-BR-3er cells being treated with scramble sh-RNA or sh-BECLIN 1 for $48 \mathrm{~h}$. (B) Cells were treated with scramble sh-RNA or sh-BECLIN 1 for $48 \mathrm{~h}$, followed by treatment with $9.6 \mathrm{nM}$ PTX for another $48 \mathrm{~h}$, then treated with $0.1 \mathrm{mM} \mathrm{MDC}$ for $1 \mathrm{~h}$, and MDC sequestration was measured with a microplate fluorometer. The OD values of MDC fluorescence (mean \pm S.E.) were from three independent experiments (PTX compared with PTX plus sh-BECLIN 1, $p=0.001$; PTX plus scramble compared with PTX plus sh-BECLIN 1, $p=0.001$ ). (C) Cells were treated with scramble sh-RNA or sh-BECLIN 1 for $48 \mathrm{~h}$, followed by treatment with $9.6 \mathrm{nM}$ PTX plus or minus $20 \mu \mathrm{M} \mathrm{Z}$-VAD-fmk for $48 \mathrm{~h}$. Cell viability was measured with trypan blue exclusion. The number of viable cells (mean \pm S.E.) was derived from three independent experiments, and the results are expressed as the relative percentage of each group compared to control (PTX alone compared with PTX plus sh-BECLIN 1, $p=0.001$; PTX plus sh-BECLIN 1 compared with PTX plus sh-BECLIN 1 and Z-VAD-fmk, $p=0.002$; MCF-7 with PTX compared to MCF-7er with PTX plus sh- $A T G 7, p=0.042)$. (D) Cleaved caspase-9 was detected with western blot after SK-BR-3er cells being treated with scramble sh-RNA or sh-BECLIN 1 for 48 h, followed by treatment with $9.6 \mathrm{nM}$ PTX for $48 \mathrm{~h}$. 


\title{
Supplementary Information
}

\section{Autophagy facilitates multidrug resistance development through inhibition of apoptosis in breast cancer cells}

\author{
W. L. SUN*, D. LAN, T. Q. GAN, Z. W. CAI
}

Department of Internal Medicine-Oncology, The First Affiliated Hospital, Guangxi Medical University, Nanning, Guangxi, China, 530021

*Correspondence: swl20022001@hotmail.com

Supplementary Figure

SK-BR-3
$\downarrow$
0.06
$\downarrow$
0.075
$\downarrow$
0.1
$\downarrow$
0.125
$\downarrow$
0.15
$\downarrow$
0.225
$\downarrow$
0.35
$\downarrow$
0.525
$\downarrow$
0.8
$\downarrow$
1.2
$\downarrow$
1.8
$\downarrow$
2.7
$\downarrow$
4.0
$\downarrow$
6.0
$\downarrow$
7.5
$\downarrow$
9.0
$\downarrow$
SK-BR-3er

Figure S1. Stepwise drug selection to establish EPI-resistant SK-BR-3 cells (SK-BR-3er cells). The dose of EPI began with $0.06 \mu \mathrm{M}$ (1/50 IC50, IC50: $3.0 \mu \mathrm{M}$ in SK-BR-3 cells), and it was increased by a dose gradient that was $25 \sim 50 \%$ of the previous dose. SK-BR-3er cells could tolerate $9.0 \mu \mathrm{M}$ EPI. During the stepwise drug selection process, cells were not clonal selected.

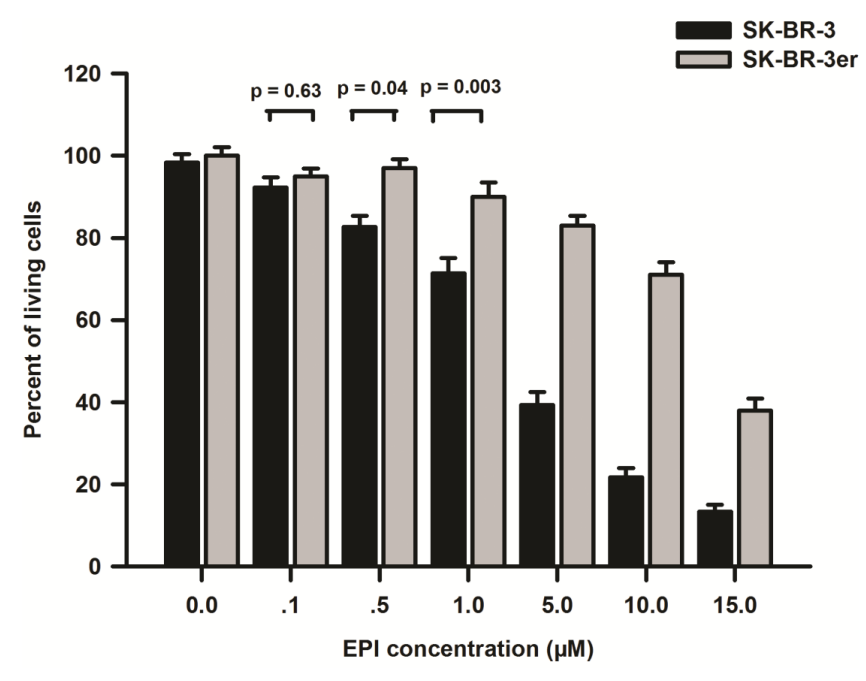

Figure S2. IC50 of SK-BR-3 cells and SK-BR-3er cells to EPI. SK-BR-3 cells and SK-BR-3er cells were treated with a different concentrations of EPI for $48 \mathrm{~h}$, and the proliferation rate was assayed with MTT. The results (mean \pm S.E.) were from three independent experiments. 
A

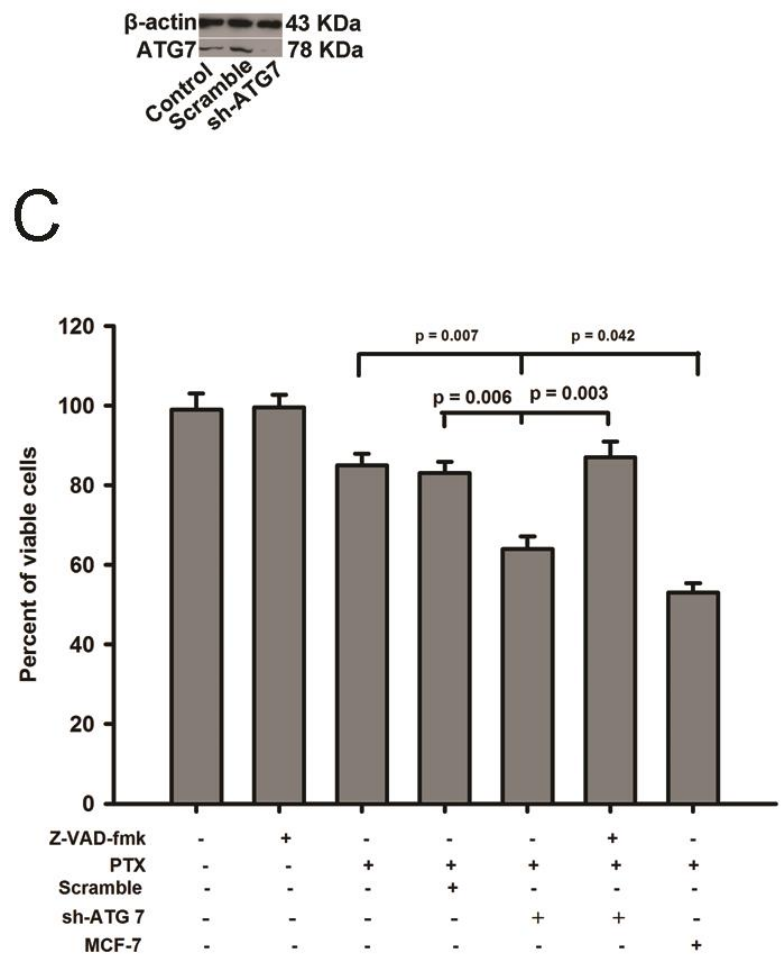

B

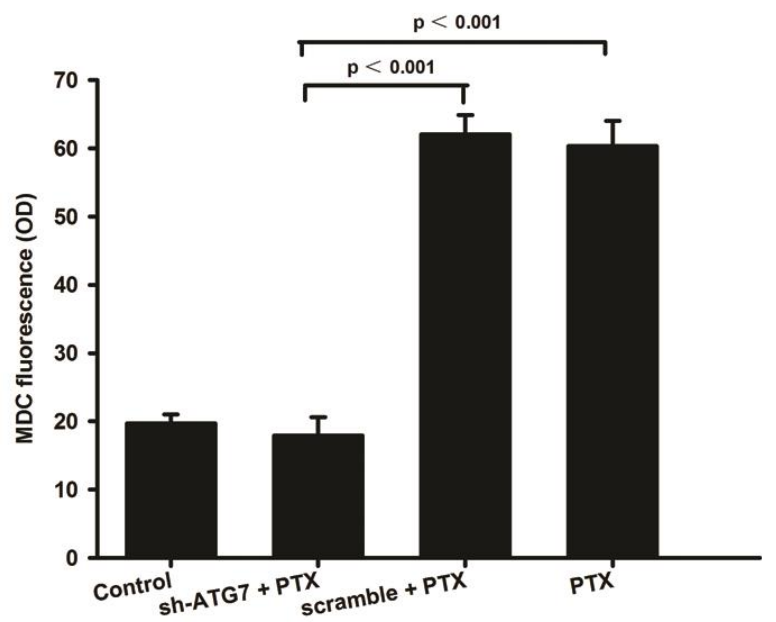

D

Figure S3. Inhibition of autophagy by sh-ATG7 increased the sensitivity of MCF-7er cells to PTX. (A) Atg7 was detected with western blot after MCF-7er cells being treated with scramble sh-RNA or sh-ATG7 for $48 \mathrm{~h}$. (B) Cells were treated with scramble sh-RNA or sh-ATG7 for 48 h, followed by treatment with $8.5 \mathrm{nM}$ PTX for another $48 \mathrm{~h}$, then treated with $0.1 \mathrm{mM}$ MDC for $1 \mathrm{~h}$, and MDC sequestration was measured with a microplate fluorometer. The OD values of MDC fluorescence (mean \pm S.E.) were from three independent experiments (PTX compared with PTX plus sh-ATG7, $p<0.001$; PTX plus scramble compared with PTX plus sh-ATG7, $p<0.001)$. (C) Cells were treated with scramble sh-RNA or sh-ATG7 for $48 \mathrm{~h}$, followed by treatment with $8.5 \mathrm{nM}$ PTX plus or minus $20 \mu \mathrm{M}$ Z-VAD-fmk for $48 \mathrm{~h}$. Cell viability was measured with trypan blue exclusion. The number of viable cells (mean \pm S.E.) was derived from three independent experiments, and the results are expressed as the relative percentage of each group compared to control (PTX alone compared to PTX plus sh-ATG7, P = 0.007; PTX plus sh-ATG7 compared with PTX plus sh-ATG7 and Z-VAD-fmk, $p=0.003$; MCF-7 with PTX compared to MCF-7er with PTX plus sh-ATG7, $p=0.042$ ). (D) Cleaved caspase-9 was detected with western blot after MCF-7er cells being treated with scramble sh-RNA or sh-ATG7 for $48 \mathrm{~h}$, followed by treatment with $8.5 \mathrm{nM}$ PTX for $48 \mathrm{~h}$. 

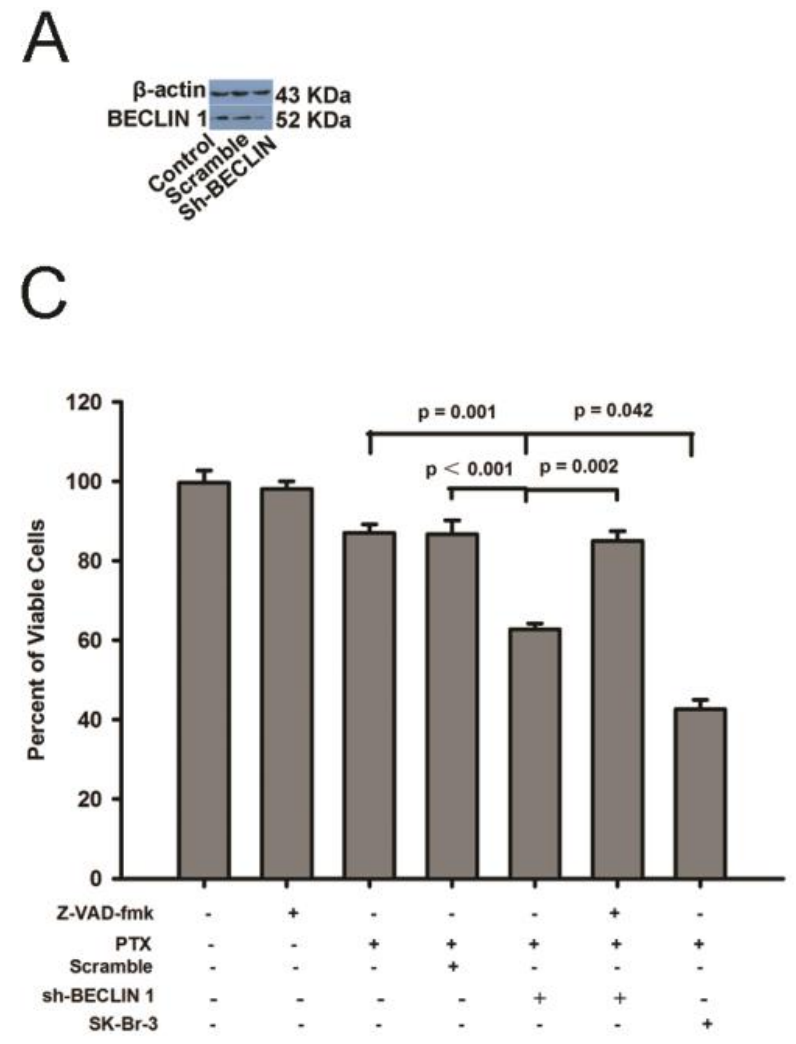
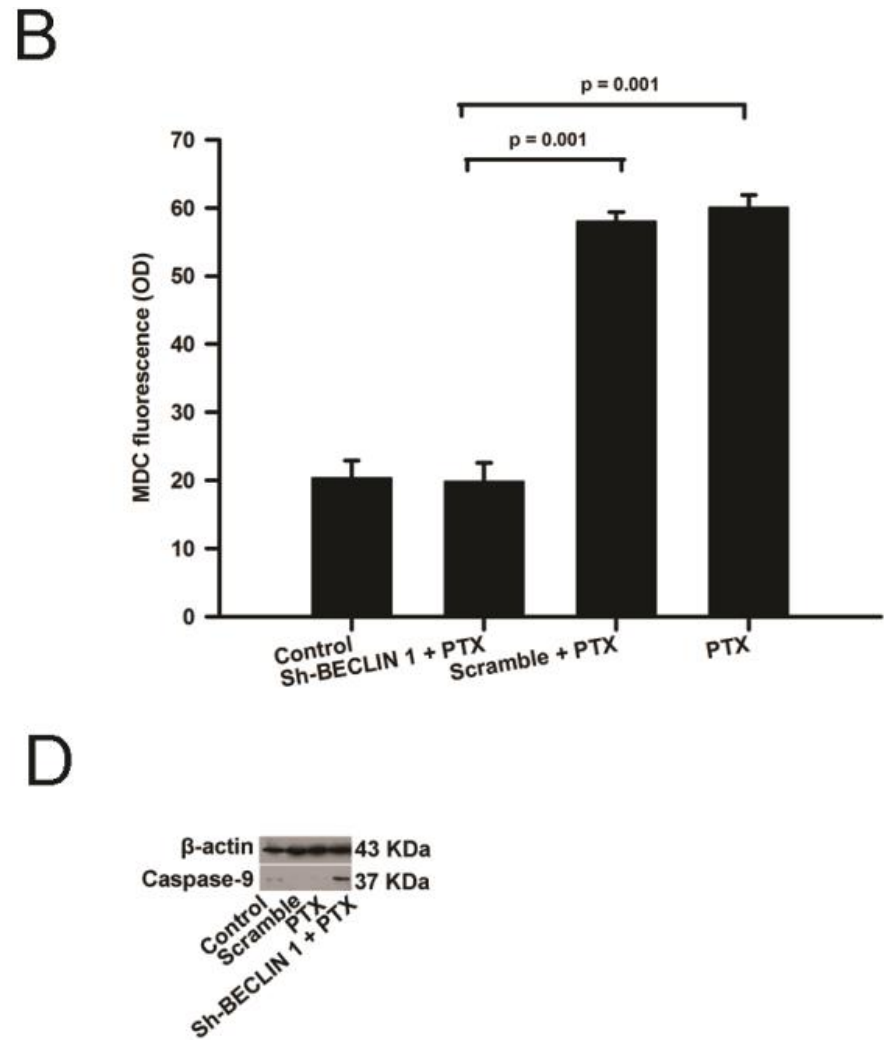

Figure S4. Inhibition of autophagy by sh-BECLIN 1 increased the sensitivity of SK-BR-3er cells to PTX. (A) Beclin 1 was detected with western blot after SK-BR-3er cells being treated with scramble sh-RNA or sh-BECLIN 1 for $48 \mathrm{~h}$. (B) Cells were treated with scramble sh-RNA or sh-BECLIN 1 for $48 \mathrm{~h}$, followed by treatment with $9.6 \mathrm{nM}$ PTX for another $48 \mathrm{~h}$, then treated with $0.1 \mathrm{mM} \mathrm{MDC}$ for $1 \mathrm{~h}$, and MDC sequestration was measured with a microplate fluorometer. The OD values of MDC fluorescence (mean \pm S.E.) were from three independent experiments (PTX compared with PTX plus sh-BECLIN 1, $p=0.001$; PTX plus scramble compared with PTX plus sh-BECLIN 1, $p=0.001$ ). (C) Cells were treated with scramble sh-RNA or sh-BECLIN 1 for $48 \mathrm{~h}$, followed by treatment with $9.6 \mathrm{nM}$ PTX plus or minus $20 \mu \mathrm{M} \mathrm{Z}$-VAD-fmk for $48 \mathrm{~h}$. Cell viability was measured with trypan blue exclusion. The number of viable cells (mean \pm S.E.) was derived from three independent experiments, and the results are expressed as the relative percentage of each group compared to control (PTX alone compared with PTX plus sh-BECLIN 1, $p=0.001$; PTX plus sh-BECLIN 1 compared with PTX plus sh-BECLIN 1 and Z-VAD-fmk, $p=0.002$; MCF-7 with PTX compared to MCF-7er with PTX plus sh- $A T G 7, p=0.042)$. (D) Cleaved caspase-9 was detected with western blot after SK-BR-3er cells being treated with scramble sh-RNA or sh-BECLIN 1 for 48 h, followed by treatment with $9.6 \mathrm{nM}$ PTX for $48 \mathrm{~h}$. 